\title{
Magnetoliposomes based on manganese ferrite nanoparticles as nanocarriers for antitumor drugs
}

\author{
Ana Rita O. Rodrigues, ${ }^{1}$ José M. F. Ramos, ${ }^{1}$ I. T. Gomes, ${ }^{1,2}$ Bernardo G. Almeida, ${ }^{1}$ J. P. Araújo, ${ }^{2}$ \\ Maria João R. P. Queiroz, ${ }^{3}$ Elisabete M. S. Castanheira, ${ }^{1}$ Paulo J. G. Coutinho ${ }^{1}$ \\ ${ }^{1}$ Centre of Physics (CFUM), University of Minho, Campus de Gualtar, 4710-057 Braga, Portugal \\ 2 IFIMUP/IN - Institute of Nanoscience and Nanotechnology, University of Porto, R. Campo Alegre, \\ 4169-007 Porto, Portugal \\ ${ }^{3}$ Centre of Chemistry (CQ-UM), University of Minho, Campus de Gualtar, 4710-057 Braga, Portugal \\ ritarodrigues@fisica.uminho.pt
}

Guided transport of biologically active molecules to target specific sites in human body has been a focus of the research in therapeutics in the past few years. Magnetoliposomes (liposomes entrapping magnetic nanoparticles) are of large importance, as they can overcome pharmacokinetics problems of the encapsulated drugs and can be guided and localized to the therapeutic sites of interest by external magnetic field gradients $[1,2]$. The use of magneto-sensitive liposomes as nanocarriers allows a safer use of powerful anticancer drugs in therapy with lower drug dosage and a more efficient treatment, not only in cancer but also in other diseases.

In this work, manganese ferrite $\left(\mathrm{MnFe}_{2} \mathrm{O}_{4}\right)$ nanoparticles with superparamagnetic behaviour at room temperature and size distribution of $26 \pm 5 \mathrm{~nm}$, were obtained by coprecipitation method. Structural and magnetic properties of the nanoparticles (NPS) were evaluated by XRD, HR-TEM and SQUID. The synthesized NPs were either entrapped in liposomes, originating aqueous magnetoliposomes (AMLs), or covered with a lipid bilayer, forming solid magnetoliposomes (SMLs), the latter prepared by a new method, recently developed for magnetoliposomes based on nickel ferrite NPs [3]. The resulting liposomes exhibit sizes below $150 \mathrm{~nm}$ (Fig. 1), suitable for biomedical applications.

Membrane fusion between both types of magnetoliposomes and GUVs (giant unilamellar vesicles), used as models of cell membranes, was confirmed by FRET (Förster Resonance Energy Transfer) assays [3-5]. For that purpose, the labeled lipid NBD-C ${ }_{12}-\mathrm{HPC}$ and the hydrophobic probe Nile Red (or the labeled lipid Rhodamine B-DOPE) were both incorporated in the lipid bilayer of magnetoliposomes, the NBD moiety acting as the energy donor and the dye Nile Red (or Rhodamine B) as the energy acceptor (Fig. 2). After interaction with GUVs, an increase in the NBD (donor) emission band and a decrease of acceptor fluorescence is observed (Fig. 2A), confirming membrane fusion (Fig. 2B) [5].

A new potential antitumor drug, a thienopyridine derivative (Fig. 3), was successfully incorporated in the lipid bilayer of both types of magnetoliposomes. This thienopyridine derivative presents very low growth inhibitory concentration values $\left(\mathrm{Gl}_{50}\right)$, between 3.5 and $6.9 \mu \mathrm{M}$, when tested in vitro against several human tumor cell lines, namely MCF-7 (breast adenocarcinoma), A375-C5 (melanoma) and NCl-H460 (non-small cell lung cancer) and was the most active of a series of analogues [6]. Moreover, this compound has shown a very low affinity for the multidrug resistance protein MDR1 [7], being suitable as an anticancer agent.

These results point to a promising application of magnetoliposomes in oncological therapy, simultaneously as hyperthermia agents and as nanocarriers for antitumor drugs, taking also advantage of magnetic directioning.

\section{References}

[1] A. S. Lubbe, C. Bergemann, J. Brock, D. G. McClure, J. Magn. Magn. Mat. 194 (1999) 149-155.

[2] S. Dandamudi, R. B. Campbell, Biomaterials 28 (2007) 4673-4683.

[3] A. R. O. Rodrigues, I. T. Gomes, B. G. Almeida, J. P. Araújo, E. M. S. Castanheira, P. J. G. Coutinho, Phys. Chem. Chem. Phys. 17 (2015) 18011-18021.

[4] A. R. O. Rodrigues, I. T. Gomes, B. G. Almeida, J. P. Araújo, E. M. S. Castanheira, P. J. G. Coutinho, Mat. Chem. Phys. 148 (2014) 978-987. 
[5] A. R. O. Rodrigues, J. M. F. Ramos, I. T. Gomes, B. G. Almeida, J. P. Araújo, M.-J. R. P. Queiroz, E. M. S. Castanheira, P. J. G. Coutinho, submitted to publication.

[6] M.-J. R. P. Queiroz, R. C. Calhelha, L. Vale-Silva, E. Pinto, M. S.-J. Nascimento, Eur. J. Med. Chem. 45 (2010) 5732-5738.

[7] C. N. C. Costa, A. C. L. Hortelão, J. M. F. Ramos, A. D. S. Oliveira, R. C. Calhelha, M.-J. R. P. Queiroz, P. J. G. Coutinho, E. M. S. Castanheira, Photochem. Photobiol. Sci. 13 (2014) 1730-1740.

\section{Figures}

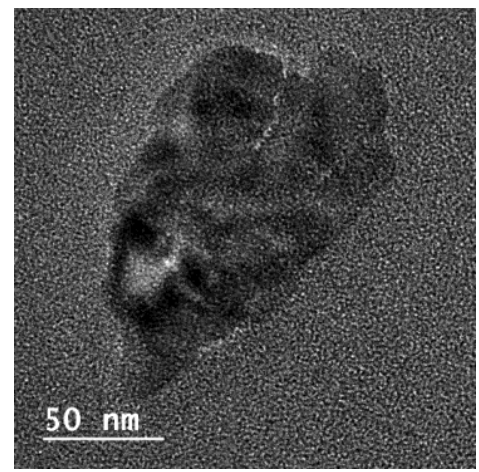

Figure 1. TEM image of solid magnetoliposomes (SMLs) containing $\mathrm{MnFe}_{2} \mathrm{O}_{4} \mathrm{NPs}$.

A

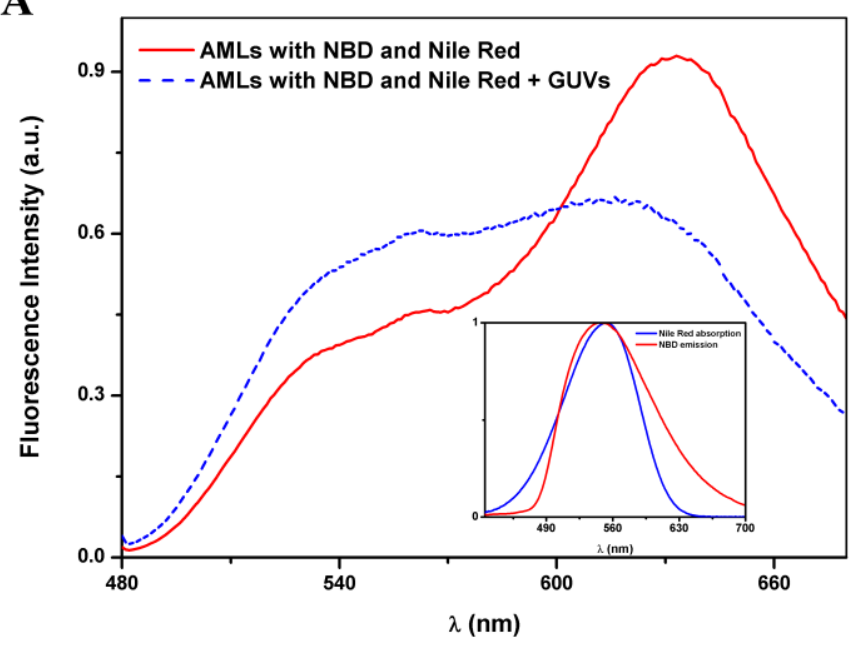

\section{B}

BEFORE MEMBRANE FUSION

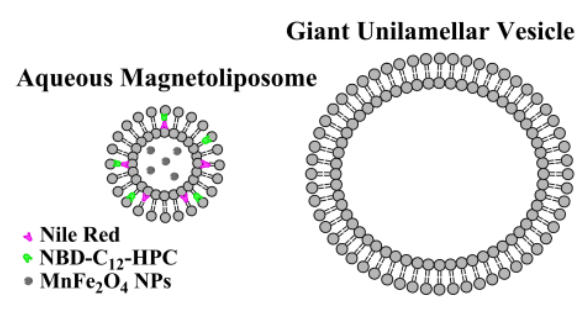

AFTER MEMBRANE FUSION

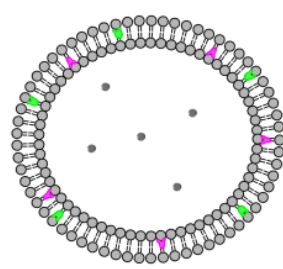

Figure 2. A. Fluorescence spectra $\left(\lambda_{e x c}=400 \mathrm{~nm}\right)$ of AMLs containing both NBD- $\mathrm{C}_{12}-\mathrm{HPC}$ and Nile Red, before and after interaction with GUVs. Inset: Spectral overlap between the fluorescence emission of the donor (NBD-C $\mathrm{C}_{12}-\mathrm{HPC}$ ) and the absorption of the acceptor (Nile Red). B. Schematic representation of membrane fusion between AMLs and GUVs.<smiles>CC(=O)c1sc2cc(Nc3nc4ccccc4s3)cnc2c1N</smiles>

Figure 3. Structure of the antitumor thienopyridine derivative. 